operation $E$ may be required twice for a singly degenerate matrix:

$$
A=\left(\begin{array}{lll}
1 & 0 & 0 \\
0 & 0 & 1 \\
0 & 0 & 0
\end{array}\right)
$$

Westinghouse Electric Corporation

Baltimore, Maryland

1. H. Garner, "The residue number system," IRE Trans. on Electronic Computers, Vol. EC-8, pp 140-147, June 1959.

2. Frazer, Duncan \& Collar, Elementary Matrices, Cambridge University Press, 1960.

\title{
A Remark on a Paper of Bateman and Horn
}

\section{By A. Schinzel}

Let $f_{1}, f_{2}, \cdots, f_{k}$ be distinct irreducible polynomials with integral coefficients and the highest coefficient positive, such that $f(x)=f_{1}(x) f_{2}(x) \cdots f_{k}(x)$ has no fixed divisor $>1$. Denote by $P(N)$ the number of positive integers $x \leqq N$ such that all numbers $f_{1}(x), f_{2}(x), \cdots, f_{k}(x)$ are primes.

P. T. Bateman and R. A. Horn [1] recently gave the heuristic asymptotic formula for $P(N)$ :

$$
P(N) \sim \frac{N}{\log ^{k} N}\left(h_{1} h_{2} \cdots h_{k}\right)^{-1} \prod_{p}\left(1-\frac{\omega(p)}{p}\right)\left(1-\frac{1}{p}\right)^{-k},
$$

where $h_{i}$ is the degree of $f_{i}$ and $\omega(p)$ is the number of solutions of the congruence $f(x) \equiv 0(\bmod p)$.

Formula (1) contains as particular cases six conjectures from a well-known paper of Hardy and Littlewood [3] called by the latter Conjectures B, D, E, F, $\mathrm{K}, \mathrm{P}$, as well as their conditional theorem $\mathrm{X} 1$. This is evident except for Conjecture $\mathrm{D}$ which concerns the number of solutions of the equation

$$
a p-b p^{\prime}=k \quad(a>0, b>0,(a, b)=1)
$$

in primes $p, p^{\prime}$ with $p \leqq n$. In order to apply formula (1) here one should put $f_{1}(x)=u_{0}+b x, f_{2}(x)=v_{0}+a x, N=\frac{n-u_{0}}{b}$, where $u_{0}, v_{0}$ are fixed integers such that $a u_{0}-b v_{0}=k$.

Conjectures denoted by Hardy and Littlewood by J, M, N are of distinctly different character; besides the first has been proved by S. Chowla [2] and Ju. V. Linnik [4]. Conjecture A, (a strong form of Goldbach's Conjecture), is a particular case of $\mathrm{C}$, Conjectures $\mathrm{H}$ and $\mathrm{I}$ are particular cases of $\mathrm{G}$. It remains therefore to consider Conjectures $\mathrm{C}, \mathrm{G}, \mathrm{L}$, which are, according to Hardy and Littlewood, conjugate to Conjectures $\mathrm{D}, \mathrm{F}, \mathrm{K}$ respectively. We quote them below for the convenience of a reader, with slight changes in the notation (e.g. $p, p^{\prime}$ denote primes).

Received February 4, 1963, revised May 13, 1963. 
Conjecture C. If $a, b$ are fixed positive integers and $(a, b)=1$ and $P(k)$ is the number of representations of $k$ in the form

$$
k=a p+b p^{\prime}
$$

then

$$
P(k)=o\left(\frac{k}{(\log k)^{2}}\right)
$$

unless $(k, a)=1,(k, b)=1$, and one and only one of $k, a, b$ is even. But if these conditions are satisfied then

$$
P(k) \sim \frac{2 C_{2}}{a b} \frac{k}{(\log k)^{2}} \prod\left(\frac{p-1}{\mathfrak{p}-2}\right),
$$

where

$$
C_{2}=\prod_{p=3}^{\infty}\left(1-\frac{1}{(p-1)^{2}}\right)
$$

and the first product extends over all odd primes $p$ which divide $k, a$ or $b$.

Conjecture G. Suppose that $a$ and $b$ are integers, and $a>0$, and let $P(n)$ be the number of representations of $n$ in the form $a m^{2}+b m+p$. Then if $n, a, b$ have $a$ common factor, or if $n$ and $a+b$ are both even, or if $b^{2}+4 a n$ is a square then

$$
P(n)=o\left(\frac{\sqrt{n}}{\log n}\right) \text {. }
$$

In all other cases

$$
P(n) \sim \frac{\epsilon}{\sqrt{a}} \frac{\sqrt{n}}{\log n} \prod\left(\frac{\mathfrak{p}}{\mathfrak{p}-1}\right) \prod_{\substack{p \geqq 3 \\ p \nmid \chi^{3}}}\left(1-\frac{1}{p-1}\left(\frac{b^{2}+4 a n}{p}\right)\right),
$$

where $\mathfrak{p}$ is a common odd prime divisor of $a$ and $b$, and $\epsilon$ is 1 if $a+b$ is odd and 2 if $a+b$ is even.

Conjecture L. Every large number $n$ is either a cube or the sum of a prime and a (positive) cube. The number $P(n)$ of representations is given asymptotically by

$$
P(n) \sim \frac{n^{\frac{1}{3}}}{\log n} \prod_{p}\left(1-\frac{1}{p-1}(n)_{p}\right),
$$

where $p \equiv 1(\bmod 3), p \nmid n$, and $(n)_{p}$ is equal to 1 or to $-\frac{1}{2}$ according as $n$ is or is not a cubic residue of $p$.

A comparison of formula (1) with the above formulas of paper [3] suggests forcibly the following conjecture.

Let polynomials $f_{1}, f_{2}, \cdots, f_{k}(k \geqq 0), f=f_{1} f_{2} \cdots f_{k}$ satisfy the same conditions as above. Let $g$ be a polynomial with integral coefficients and the highest coefficient positive. Let $n$ be a positive integer such that $n-g(x)$ is irreducible and $f(x)(n-g(x))$ has no fixed divisor $>1$. Denote by $N(n)=N$ the number of 
positive integers $x$ such that $n-g(x)>0$ and by $P(n)$ the number of $x$ 's such that all numbers $f_{1}(x), f_{2}(x), \cdots, f_{k}(x)$ and $n-g(x)$ are primes. Then for large $n$ we have

$$
P(n) \sim \frac{N}{\log ^{k+1} N}\left(h_{0} h_{1} \cdots h_{k}\right)^{-1} \prod_{p}\left(1-\frac{\omega(p)}{p}\right)\left(1-\frac{1}{p}\right)^{-k-1}
$$

where $h_{0}$ is the degree of $g$ and $\omega(p)$ is the number of solutions of the congruence $f(x)(n-g(x)) \equiv 0(\bmod p)$.

Conjectures C, G, L and therefore also A, H, I are particular cases of formula (3). To see this, as far as $\mathrm{C}$ is concerned, one should put

$$
f_{1}(x)=b x+l, \quad g(x)=a x, \quad n=\frac{k-a l}{b},
$$

where $l$ is an integer such that $a l \equiv k(\bmod b),-b<l \leqq 0$. Conjecture A has been extensively verified [3, p. 37]. I have had no possibility to verify by computation the agreement of formula (3) with reality in other cases. For such comparisons one should replace $N(\log N)^{-k-1}$ by $\int_{2}^{N}(\log u)^{-k-1} d u$, as is pointed out in [3].

I conclude with expressing my thanks to the referee for his valuable suggestions.

Mathematics Institute PAN

Sniadeckich 8, Warsaw 1

Poland

1. P. T. Bateman \& R. A. Horn, "A heuristic asymptotic formula concerning the distribution of prime numbers," Math. Comp., v. 16, 1962, p. 363-367.

2. S. CHOwlA, "The representation of a number as a sum of four squares and a prime," Acta Arith., v. 1, 1935, p. 115-122.

3. G. H. HARDY \& J. E. LitTlewood, "Some problems of 'Partitio numerorum'; III: On the expression of a number as a sum of primes," Acta Math., v. 44, 1923, p. 1-70.

4. JU. V. LiNNIK, "An asymptotic formula in an additive problem of Hardy-Littlewood," (Russian), Izv. Akad. Nauk SSSR. Ser. Mat., v. 24, 1960, p. 629-706.

\section{Some Miscellaneous Factorizations}

\section{By John Brillhart}

1. Introduction. The factorizations presented here have accumulated in the author's files for several years and have not heretofore appeared in print. Twentyfive contain new prime factors (designated by an asterisk), while twenty-one, listed as complete, possess a cofactor that is prime. Included are the complete factorizations of four Mersenne numbers and twelve Fibonacci numbers. Further results include a second factor of the Mersenne number $M_{191}$ and of the Fermat number $F_{10}$, as well as the least prime factor of $M_{271}$. These new results relating to Mersenne numbers supplement an earlier tabulation by G. D. Johnson and the author [1].

The factorizations, with the exception of those numbered (6)-(9), inclusive, were obtained by the author on various IBM computers at the University of California at Berkeley and at Los Angeles. The factorizations (6)-(9) of certain alge-

Received January 3, 1963, revised March 12, 1963. 